\title{
Position Problem
}

National Cancer Institute

\section{Source}

National Cancer Institute. Position Problem. NCI Thesaurus. Code C63034.

Problem associated with the movement of the device to an intended location. 